\title{
Prioritization by transients in visual search
}

\author{
ARTEM V. BELOPOLSKY \\ University of Illinois at Urbana-Champaign, Urbana, Illinois \\ JAN THEEUWES \\ Vrije Universiteit, Amsterdam, The Netherlands \\ and \\ ARTHUR F. KRAMER \\ University of Illinois at Urbana-Champaign, Urbana, Illinois
}

\begin{abstract}
There is an ongoing debate as to whether prioritizing new objects over old objects (the so-called preview benefit) is the result of top-down inhibition of old objects (i.e., visual marking; Watson \& Humphreys, 1997) or attentional allocation to new objects, presented with a luminance transient (Donk \& Theeuwes, 2001). In the two experiments reported here, we tested whether prioritization by luminance transients alone can produce a subset-selective search similar to the preview effect. Subjects viewed multiobject displays while a subset of objects was briefly flashed. The subjects prioritized up to 14 flashed objects over at least 14 nonflashed objects. Since prioritization by luminance transients can produce a subsetselective search on its own, it may well play an important role in the preview benefit.
\end{abstract}

The visual system utilizes a variety of attentional mechanisms to prioritize the selection of relevant information in the environment. Recently, Watson and Humphreys (1997) showed that visual search can be significantly improved when the presentation of search objects is separated in time. If a subset of distractors is previewed for at least $400 \mathrm{msec}$ before the search display, the previewed objects can be excluded from search. This phenomenon, termed the preview effect, appears to be fairly robust across a variety of conditions; it occurs with previewed subsets of up to 15 objects (Theeuwes, Kramer, \& Atchley, 1998) and is not affected if search objects change color (Watson \& Humphreys, 1997, 2002). Watson, Humphreys, and colleagues (Watson \& Humphreys, 1997, 2002) argued that the preview effect is a result of top-down inhibition of the previewed (old) objects, a mechanism that they termed visual marking.

Even though top-down inhibition represents the modal view of the mechanism underlying the preview benefit, recently Donk and Theeuwes (2001) proposed an alternative account. They suggested that the preview effect occurs as a result of the prioritization of new objects (Yantis \& Jones, 1991). They showed that the preview effect critically depends on whether the new objects are ac-

This research was supported by grants from the National Institute on Aging (RO1 AG18008) and the General Motors Corporation. We thank Yuhong Jiang, Derrick Watson, Glyn Humphreys, and Cathleen Moore for their excellent comments and suggestions on earlier drafts of this article. We also thank Shawn Bolin, Russell Smith, and Ensar Becic for their assistance in running the subjects. Correspondence regarding this article should be addressed to A. V. Belopolsky, Beckman Institute, University of Illinois, 405 N. Mathews Ave., Urbana, IL 61801 (e-mail: belopols@ uiuc.edu). companied by luminance increments. When the new objects were presented as isoluminant with the background, the preview effect was abolished.

Even though Donk and Theeuwes's (2001) account seems viable, several arguments in favor of the top-down inhibitory account of visual marking have been put forward. First, the preview effect seems to have a relatively long time course; old objects have to be previewed for at least $400 \mathrm{msec}$ in order for the preview effect to emerge, and it reaches asymptote at about 1,000 msec. Second, the preview effect is reduced if subjects are engaged in a demanding secondary task during the preview (Olivers \& Humphreys, 2002; Watson \& Humphreys, 1997). This implies that inhibition is an active top-down process that can be disrupted by a secondary task. Third, probe detection studies have shown that the detection of a probe dot was the worst at the locations of old objects, suggesting active inhibition (Watson \& Humphreys, 2000). Finally, it is the accepted view in the literature that only a limited number of luminance transients, about four, can be prioritized for search (Burkell \& Pylyshyn, 1997; Yantis \& Jones, 1991). In visual marking studies, it has been demonstrated that at least up to 15 new locations can be prioritized for selection (Donk \& Theeuwes, 2001; Theeuwes et al., 1998).

Given the literature reviewed above, the fundamental question as to whether the preview benefit is the result of top-down inhibition of old objects or of attentional allocation to new objects, accompanied by luminance transients, is still undecided. The experiments we report here were designed to test whether prioritization by luminance transients alone could produce a subset-selective search similar to the one found in the preview paradigms. 
We designed a paradigm in which a search subset was defined solely on the basis of luminance transients and there was no preview of a subset of objects. Thus, there were no old or new objects, and inhibition would be an unlikely strategy. In order to assess search through the flashed and the nonflashed subsets independently, the two different sets of objects (i.e., 6, 10, and 14 flashed and 6, 10, and 14 nonflashed) were orthogonally manipulated (see Theeuwes et al., 1998, for a similar manipulation). The major difference from the preview search paradigm was that all the objects were presented simultaneously and then a search subset (containing the target) was designated by a brief luminance change. To ensure that the onset of the display was not a cue to start searching, we presented all the stimuli on the screen before the subject knew the identity of the target. Simultaneously with the flash, the identity of the target that the subjects had to search for was revealed by playing a sound file. If attentional allocation is limited to four simultaneous locations (Burkell \& Pylyshyn, 1997; Yantis \& Jones, 1991), subjects should not be able to search flashed objects exclusively and would have to sample a large number of nonflashed objects as well. This would mean that prioritization by onsets can only partially account for the preview benefit, and a dominant role of other mechanisms, such as inhibition, would have to be assumed. However, if attention can be allocated to multiple locations defined by luminance transients, the subjects should be able to restrict their search to a flashed subset of objects. Such a finding would mean that prioritization by onsets might play an important role in the preview effect. This hypothesis was tested in Experiment 1.

However, even if subjects are able to restrict their search to the flashed subset, it does not necessarily mean that no active processing is devoted to the nonflashed objects. There is a chance that other active mechanisms, such as maintaining attention or inhibiting the nonflashed subset, in order to segregate it from the flashed subset, could also work together with prioritization by luminance transients to produce subset-selective search. This issue was addressed in Experiment 2.

\section{EXPERIMENT 1}

The goal of this experiment was to test whether prioritization by luminance transients can produce a subsetselective search similar to the one found in the preview search paradigms. If prioritization is restricted to about four objects, search times should be largely dependent on the numbers of both flashed and nonflashed objects. However, if exclusive prioritization of multiple locations is possible, search times should be independent of the number of nonflashed objects and should depend only on the number of flashed objects.

\section{Method}

Subjects. Ten students from the University of Illinois, between 18 and 26 years old (average age, 22 years; 5 males) were paid to participate in the experiment. All the subjects had normal or correctedto-normal visual acuity and were native speakers of English.

Apparatus. A Micron 166-MHz computer, equipped with a set of headphones and running custom software, was used for stimulus generation, presentation, and recording the subjects' reaction times (RTs) and accuracy data. The stimuli were presented in 16-bit color depth on a 20 -in. SVGA monitor.

Stimuli. The stimuli were randomly sampled from all the letters of the alphabet, except for I, N, Q, T, Y, and W. They were 1 pixel wide and approximately $0.5^{\circ}$ in width and $1^{\circ}$ in height and were painted in gray $\left(12 \mathrm{~cd} / \mathrm{m}^{2}\right)$ against a black background. On each trial, the objects' locations were randomly sampled from 36 possible locations along the circumference of two concentric imaginary circles in the center of the screen. The smaller circle contained 14 possible locations, and its radius was approximately $5^{\circ}$; the larger circle contained 22 possible locations, and its radius was approximately $7.5^{\circ}$. The identity of the target letter was revealed by playing a prerecorded WAVE file with a female voice pronouncing the letter. During the trial, a subset of objects underwent a transient luminance change - that is, briefly changed color to white $\left(80 \mathrm{~cd} / \mathrm{m}^{2}\right)$. Luminance values were measured from a solid patch of color at $60 \mathrm{~cm}$ from the monitor.

Design. On any given trial, there were 6,10 , or 14 objects that underwent a transient luminance change (flashed subset) and 6, 10, or 14 objects that did not (nonflashed subset). The target letter was present on half of the trials and, when present, always appeared in the flashed subset. Each subject completed 24 experimental blocks (864 trials), with the trial order randomized within a block.

Procedure. The time course of a trial is shown in Figure 1. The trial started with a fixation cross that was presented for $500 \mathrm{msec}$.
Fixation

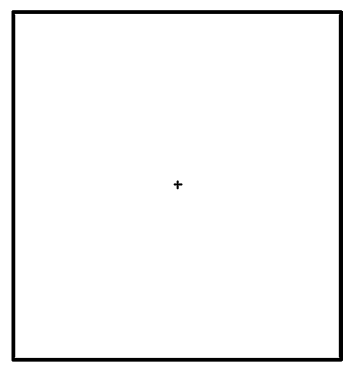

$500 \mathrm{msec}$
All Letters

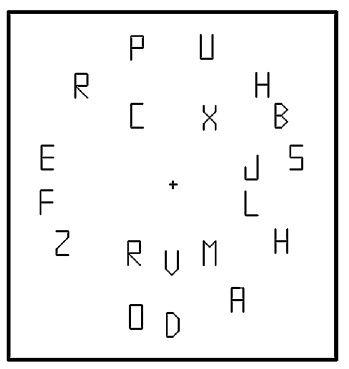

$500 \mathrm{msec}$
Flash On

Sound On: "J"

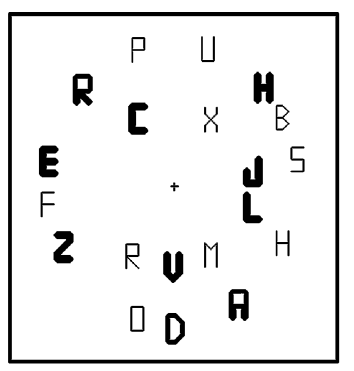

$100 \mathrm{msec}$
Flash Off

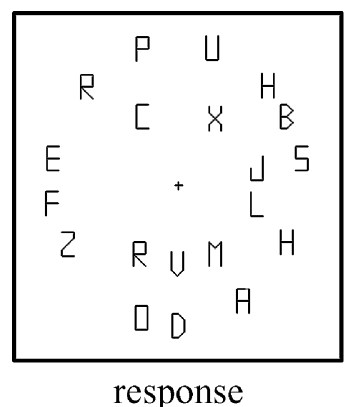

Figure 1. A graphical illustration of the displays and time course of the events in a trial with 10 flashed and 10 nonflashed objects. 
It was followed by the presentation of gray letters for another $500 \mathrm{msec}$ (this interval was used to separate the flash from the initial onset of the search display), after which a subset of objects turned white for $100 \mathrm{msec}$. The WAVE file playback started simultaneously with the onset of the flash. The stimuli stayed on the screen until a response had been made.

The subjects were seated $60 \mathrm{~cm}$ from the monitor and were told to determine the presence or absence of a target letter, spoken over the headphones. They were instructed to keep their eyes on the fixation cross (which was on the screen throughout the entire trial) until they heard a voice pronouncing the target letter. The subjects were told that on each trial a subset of letters would be flashed and that the target, when present, would always appear in the flashed subset. Half of the subjects responded with the " $z$ " key when the target was absent and with the "/" key when it was present. The response keys were reversed for the other half of the subjects. Before the start of the experiment, the subjects completed a practice block of 20 trials.

\section{Results}

RTs twice the size of a cell mean or smaller than $100 \mathrm{msec}$ were excluded from the analysis $(1.3 \%$ of the trials). Two subjects were excluded from the analyses because of an overall error rate exceeding $15 \%$.

Overall, the subjects responded more quickly in the target-present trials than in the target-absent trials $[F(1,7)=$ 9.26, $p<.05 ; 1,017$ and $1,629 \mathrm{msec}$, respectively]. For the target-present trials, a repeated measures analysis of variance (ANOVA) on mean RTs, with number of nonflashed objects $(6,10$, or 14) and number of flashed objects $(6,10$, or 14$)$ as factors, showed a main effect of the number of flashed objects $[F(2,14)=28.69, p<.001]$. RT was not affected by the number of nonflashed objects $[F(2,14)=1.92, p=.18]$. The slopes of the flashed and the nonflashed objects in the target-present conditions were 29.9 and $5.2 \mathrm{msec} /$ object, respectively. The interaction also was not significant $[F(4,28)=0.50, p=.74]$.

For the target-absent trials, both the number of the nonflashed and the number of flashed objects affected RT $[F(2,14)=10.37, p<.005$, and $F(2,14)=16.10$, $p<.001$, respectively]. The slopes of the flashed and the nonflashed objects in the target-absent conditions were 72.1 and $28.7 \mathrm{msec} /$ object, respectively. The interaction was not significant $[F(4,28)=0.69, p=.60]$. Figure 2 presents the mean correct RTs as a function of target presence, the number of nonflashed objects, and the number of flashed objects.

Mean error rates are presented in Table 1. The mean error rate was $9.5 \%$. The subjects made more errors in the target-present trials than in the target-absent trials $[F(1,7)=26.75, p<.005]$, suggesting that they responded more frequently that the target was absent when it was present than vice versa.

For the target-present trials, the error rates mirrored the RT results. There was a main effect of the number of flashed objects $[F(2,14)=5.0, p<.05]$ and an interaction between the numbers of flashed and nonflashed objects $[F(4,28)=3.28, p<.05]$. For target-absent trials, there was a significant interaction only of the number of flashed and the number of nonflashed objects $[F(4,28)=3.9, p<.05]$.

\section{Discussion}

The present results demonstrate that subjects are able to search up to 14 objects defined by luminance transients,
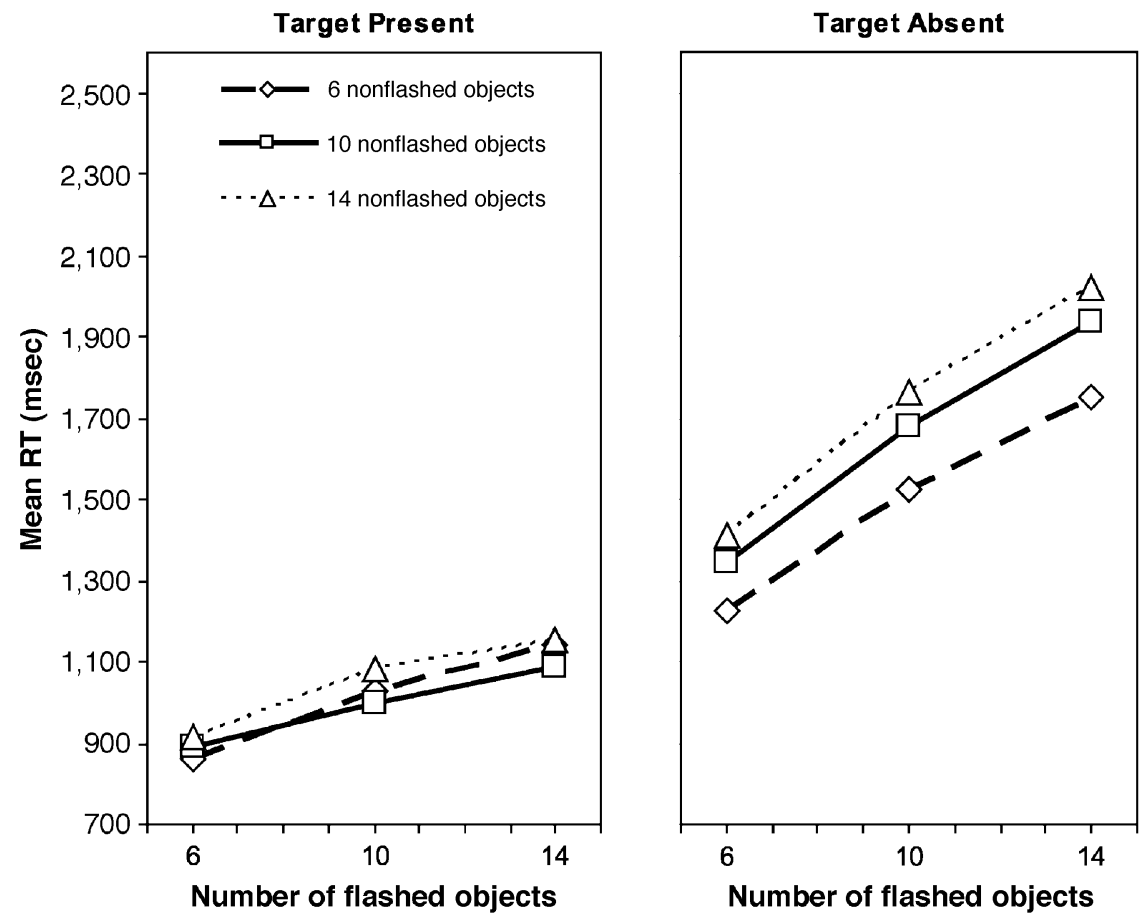

Figure 2. Mean correct reaction time (RT) as a function of target presence, the number of nonflashed objects, and the number of flashed objects in Experiment 1. 
Table 1

Mean Error Rates (in Percentages) in the Target-Present and Target-Absent Conditions in Experiment 1

\begin{tabular}{|c|c|c|c|c|c|c|c|c|c|}
\hline \multirow{4}{*}{$\begin{array}{l}\text { Experimental } \\
\text { Condition }\end{array}$} & \multicolumn{9}{|c|}{ Number of Nonflashed Objects } \\
\hline & \multicolumn{3}{|c|}{6} & \multicolumn{3}{|c|}{10} & \multicolumn{3}{|c|}{14} \\
\hline & \multicolumn{3}{|c|}{$\overline{\text { Number of Flashed Objects }}$} & \multicolumn{3}{|c|}{$\overline{\text { Number of Flashed Objects }}$} & \multicolumn{3}{|c|}{ Number of Flashed Objects } \\
\hline & 6 & 10 & 14 & 6 & 10 & 14 & 6 & 10 & 14 \\
\hline Target present & 9.9 & 14.8 & 14.6 & 16.4 & 14.1 & 18.0 & 9.4 & 16.1 & 20.3 \\
\hline Target absent & 1.3 & 3.1 & 5.7 & 5.7 & 2.9 & 3.6 & 5.2 & 4.2 & 6.0 \\
\hline
\end{tabular}

while effectively filtering out up to 14 task-irrelevant distractors. Thus, prioritization by luminance transients can produce subset-selective search and potentially plays an important role in the preview effect. However, such prioritization in our paradigm might be assisted by processing (attention or inhibition) of the nonflashed objects, relative to a situation in which all of the objects are flashed. This possibility was explored in Experiment 2.

\section{EXPERIMENT 2}

The goal of this experiment was to further examine whether there was any active processing of the nonflashed objects in a paradigm similar to that employed in Experiment 1 . This was accomplished by adding a baseline condition, in which all of the objects were flashed. Such a manipulation requires subjects to search through all of the objects in the display and provides a baseline estimate of the search rate through the flashed objects.

If we assume that active processing of nonflashed objects consumes attentional resources that otherwise would have been allocated to the selective processing of flashed objects, this would result in a significant slowing of the search rate through the flashed subset, relative to the baseline condition. However, if nonflashed objects are not actively processed, the search rate through the flashed objects should be independent of whether the subjects search through a subset of flashed objects in the presence of nonflashed objects in the experimental condition or through a full set of flashed objects in the baseline condition.

\section{Method}

Subjects. Fifteen students from the University of Illinois, between 18 and 27 years old (average age, 21 years; 7 males) were paid to participate in the experiment. All the subjects had normal or correctedto-normal visual acuity and were native speakers of English.

Apparatus and Stimuli. The equipment and stimuli were identical to those in Experiment 1. In the control condition, all of the stimuli were flashed.

Design. Two search conditions were manipulated within subjects. In the experimental condition, a subset of objects was flashed on any given trial (subset-flash), and in the control condition all of the objects in the display were flashed (all-flash). The trials in the experimental condition were identical to the trials in Experiment 1. In the control condition, the same total set size manipulations were used (12, 16, 20, 24, and 28 objects), and all of them were flashed. The same total set sizes were used in order to control for clutter across conditions. The target letter was present on half of the trials.

Experimental (subset-flash) and control (all-flash) conditions were presented in separate alternating blocks of trials ( 12 blocks per con- dition; total of 864 trials), with the trial order randomized within a block. The order of blocks was counterbalanced across subjects.

Procedure. The time course of a trial and the procedure were the same as those in Experiment 1. In the all-flash control condition, the only difference was that instead of a subset of objects, all of the objects turned white for $100 \mathrm{msec}$.

\section{Results}

RTs twice the size of a cell mean or shorter than $100 \mathrm{msec}$ were excluded from the analysis $(1.1 \%$ of the trials). Three subjects were excluded from the analyses because of an overall error rate exceeding $15 \%$. Mean correct RT is presented in Figure 3.

Repeated measures ANOVAs on mean RT for correct trials, with condition (subset-flash or all-flash), target (present or absent), and total display size $(12,16,20,24$, or 28) as factors, showed that the subjects were faster in the subset-flash condition than in the all-flash condition $[F(1,11)=39.50, p<.001 ; 1,452$ and $1,652 \mathrm{msec}$, respectively], as well as faster in the target-present trials than in the target-absent trials $[F(1,11)=53.00, p<$ $.001 ; 1,226$ and $1,878 \mathrm{msec}$, respectively].

Additional ANOVAs with the number of nonflashed objects $(6,10$, or 14) and the number of flashed objects $(6,10$, or 14$)$ as factors were performed on trials in the subset-flash condition (i.e., the same conditions as those employed in Experiment 1). In the target-present condition, RT increased only as a function of the number of flashed objects $[F(2,22)=41.05, p<.001]$ and was not significantly influenced by the number of nonflashed objects $[F(2,22)=2.67, p>.05]$. The interaction was not significant $[F(4,44)=1.54, p>.2]$. The slopes of the flashed and the nonflashed objects were 35.6 and $10.6 \mathrm{msec} /$ object, respectively. In the target-absent condition, both the number of the nonflashed and the number of the flashed objects affected RT $[F(2,22)=15.98, p<.001$, and $F(2,22)=67.51, p<.001$, respectively]. The interaction was not significant $[F(4,44)=1.86, p>.1]$. The slopes of the flashed and the nonflashed objects in the subset-flash condition were 70.7 and $35.0 \mathrm{msec} / \mathrm{object}$, respectively.

In order to compare the search rate for the flashed objects in the subset-flash and the all-flash conditions, we fitted the slopes for the function relating RTs to the number of the flashed objects $(6,10$, and 14 for the subsetflash condition and 12, 16, 20,24, and 28 for the all-flash condition) separately for each subject. For the targetpresent trials, the slopes were not significantly different 
Experimental (subset-flash)
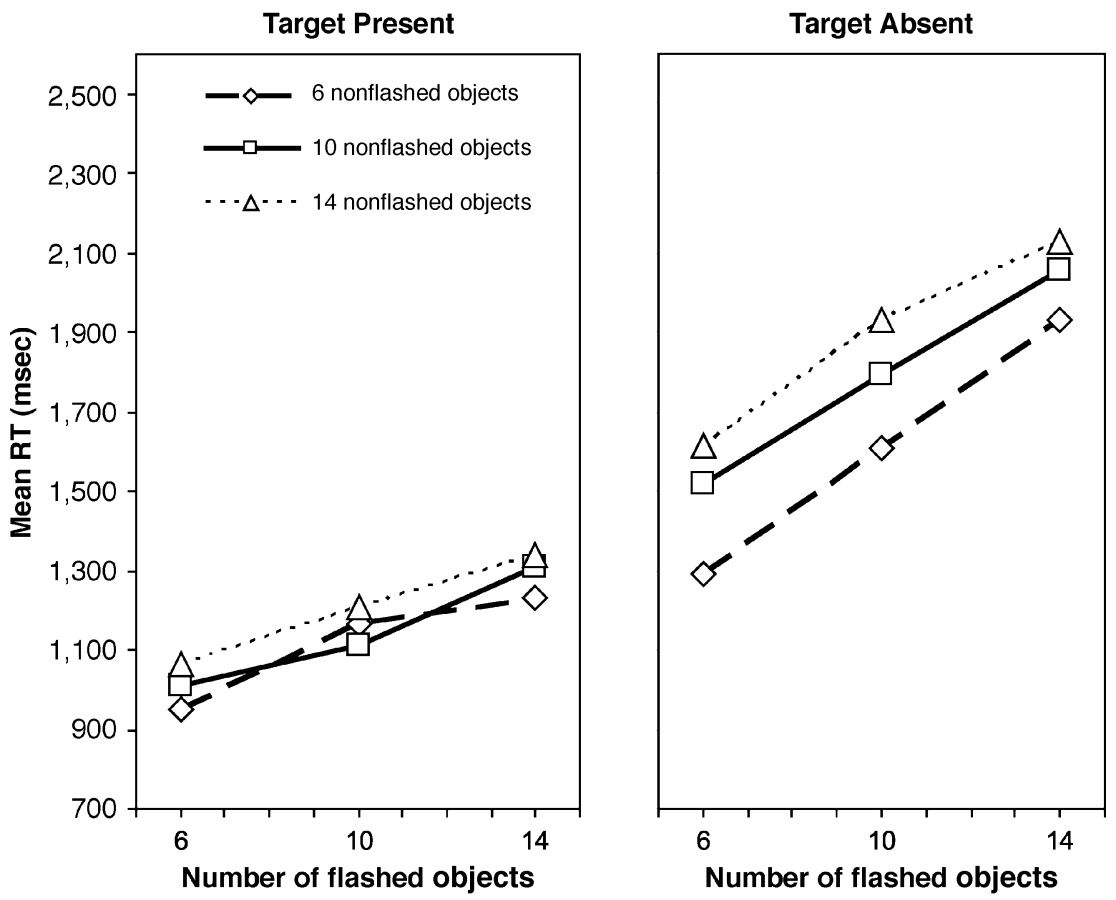

Control (all-flash)
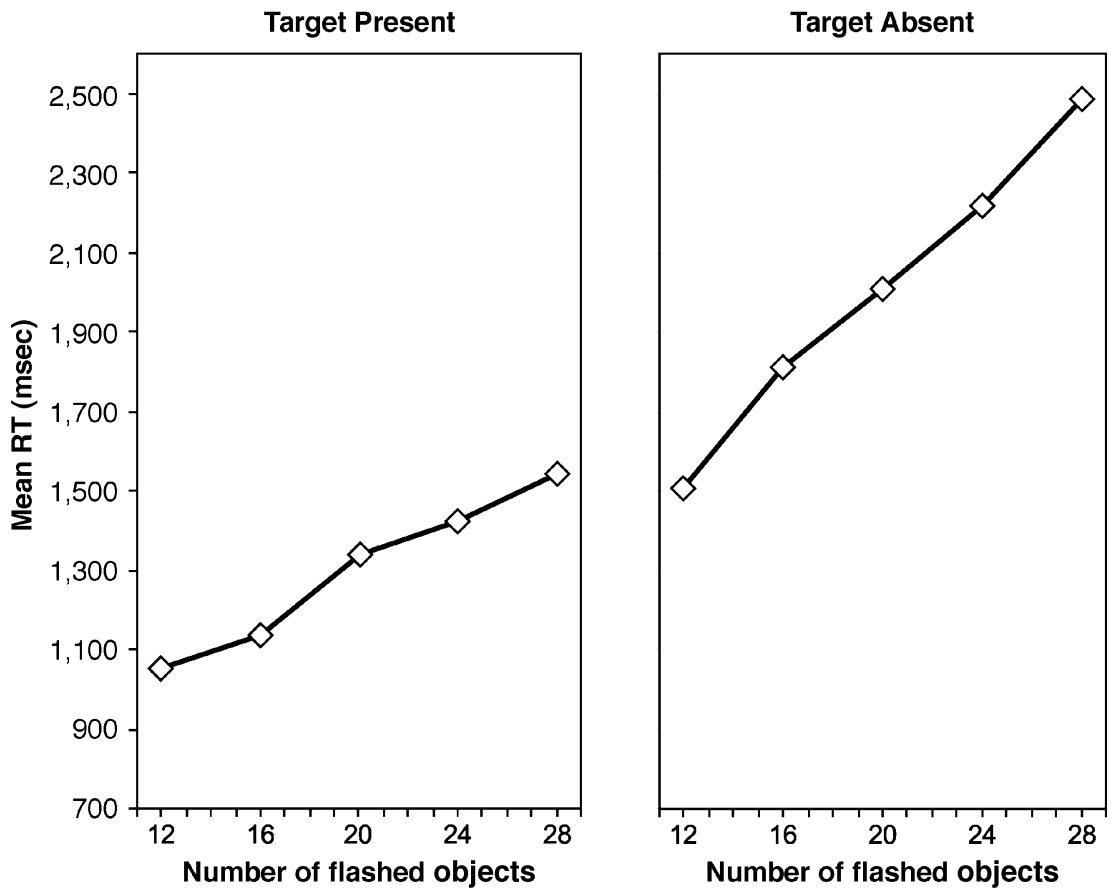

Figure 3. Mean correct reaction time (RT) as a function of target presence, the number of nonflashed objects (only in the subset-flash condition), and the number of flashed objects in the subset-flash and the all-flash conditions in Experiment 2.

[35.6 msec/object in the subset-flash condition and $31.6 \mathrm{msec} /$ object in the all-flash condition; $F(1,11)=$ $0.66, p>.4]$. For the target-absent trials, the slopes in the subset-flash condition were steeper than the slopes in the all-flash condition [70.7 and $59.1 \mathrm{msec} /$ object, respectively; $F(1,11)=8.99, p<.05]$.

Mean error rates are presented in Tables $2 \mathrm{~A}$ and $2 \mathrm{~B}$. Overall error rate was $8.7 \%$. The subjects made more er- 
Table 2A

Mean Error Rates (in Percentages) in the Experimental (Subset-Flash) Condition in Experiment 2

\begin{tabular}{|c|c|c|c|c|c|c|c|c|c|}
\hline \multirow{4}{*}{$\begin{array}{l}\text { Experimental } \\
\text { Condition }\end{array}$} & \multicolumn{9}{|c|}{ Number of Nonflashed Objects } \\
\hline & \multicolumn{3}{|c|}{6} & \multicolumn{3}{|c|}{10} & \multicolumn{3}{|c|}{14} \\
\hline & \multicolumn{3}{|c|}{ Number of Flashed Objects } & \multicolumn{3}{|c|}{ Number of Flashed Objects } & \multicolumn{3}{|c|}{ Number of Flashed Objects } \\
\hline & 6 & 10 & 14 & 6 & 10 & 14 & 6 & 10 & 14 \\
\hline Target present & 9.8 & 14.4 & 19.2 & 10.5 & 15.1 & 23.1 & 11.6 & 14.0 & 24.2 \\
\hline Target absent & 0.7 & 1.7 & 3.9 & 2.1 & 1.0 & 2.1 & 3.2 & 2.1 & 4.5 \\
\hline
\end{tabular}

Table 2B

Mean Error Rates (in Percentages) in the Control (All-Flash) Condition in Experiment 2

\begin{tabular}{lcrrrr} 
& \multicolumn{5}{c}{ Total Number of Objects } \\
\cline { 2 - 6 } Control Condition & 12 & 16 & 20 & 24 & \multicolumn{1}{c}{28} \\
\hline Target present & 8.4 & 13.3 & 11.9 & 16.3 & 16.8 \\
Target absent & 1.7 & 2.8 & 2.7 & 3.8 & 5.6 \\
\hline
\end{tabular}

rors in the target-present trials than in the target-absent trials $[F(1,11)=55.81, p<.001]$, suggesting that they tended to respond more frequently that the target was absent when it was present. Importantly, the error rate was not significantly different between the subset-flash and the all-flash conditions $[F(1,11)=1.11, p>.3]$. For the target-present trials in the subset-flash condition, there was a main effect only of the number of flashed objects $[F(2,22)=14.76, p<.001]$. Neither the number of nonflashed objects $[F(2,22)=0.86, p>.4]$ nor the interaction $[F(4,44)=0.55, p>.7]$ was significant. For targetabsent trials, none of the effects reached significance.

\section{Discussion}

The results indicate that subset-selective search based on luminance transients is not significantly slowed by the nonflashed objects, which are likely minimally processed. In addition, just as in marking studies, responses were faster in the experimental condition than in the control condition.

\section{GENERAL DISCUSSION}

Our goal was to examine whether in the absence of the ability to actively inhibit a subset of objects, the prioritization by luminance increments account proposed by Donk and Theeuwes (2001) can produce a pattern of search performance similar to that observed in the preview paradigm, in which two different sets of objects are presented at different points in time. To examine this issue, we used a different paradigm, in which the search subset was designated by a brief luminance flash. Our results demonstrate that in a visual search task, attention can be selectively allocated to multiple locations or objects (up to 14) defined by luminance transients. This number substantially exceeds the limit of 4 objects that is usually shown in studies of attentional capture or attentional object tracking (e.g., Burkell \& Pylyshyn, 1997; Yantis \& Jones, 1991). It is reasonable to assume that this was due to flashed objects being prioritized as a group. Previously, it has been demonstrated that search can be restricted to subsets, grouped by static features, such as color (Egeth, Virzi, \& Garbart, 1984; Kaptein, Theeuwes, \& van der Heijden, 1995). Note, however, that in the present study, the grouping feature was dynamic $(100 \mathrm{msec})$ and that, by the time the search began, all the items had the same physical properties.

It has recently been suggested that old and new objects can be grouped on the basis of time, and then either group could be searched exclusively at will (Jiang, Chun, $\&$ Marks, 2002). Since the flash used in our paradigm was a change over time, the present results are consistent with this account. Recently, Donk and Theeuwes (2003) showed evidence that prioritization by onsets in a preview paradigm is under bottom-up control. It is possible that prioritization by luminance transients in the present study is also bottom-up, which would suggest that it is not due to temporal grouping but, rather, to attentional capture by a group defined by luminance transients. A question for future research is whether subjects are able to exclusively (and just as quickly) search nonflashed objects when instructed to do so. Whereas the temporal segregation account predicts that this should be possible, the bottom-up attentional capture account predicts that subset-specific search should be possible only for the flashed set of objects.

Importantly, the present results closely resemble the visual-marking search data reported by Theeuwes et al. (1998), who showed that subjects selectively search through new objects and ignore old objects. Similar to Theeuwes et al., our results show that subjects first search serially and exhaustively through a set of flashed objects. If the target is not found among the flashed objects (in the case of target-absent trials), the subjects may search a few nonflashed objects and then decide that the target is not present (i.e., rechecking hypothesis; Treisman \& Gelade, 1980).

Even though it is possible that in the traditional preview paradigm, in which sets of objects are presented at different points in time, top-down inhibition may play a role in prioritization (considering evidence from probe detection studies, dual-task effects, and recent neuroimaging evidence; Olivers \& Humphreys, 2002; Pollmann et al., 2003; Watson \& Humphreys, 2000), it is 
clear that in the present experiment, in which all the objects were presented at the same time, active inhibition was unlikely. One could argue that active inhibition was applied to the nonflashed objects after the flash. However, it seems unlikely, because it is generally agreed that it takes up to $400 \mathrm{msec}$ for inhibition to accrue. Since the sound designating the target was played simultaneously with the 100-msec flash, the subjects could start searching immediately. Finally, Experiment 2 refutes the possibility of any active mechanisms assisting attentional capture through the processing of nonflashed objects by demonstrating that nonflashed objects have no significant impact on the rate of search through flashed objects.

In conclusion, we have shown that multiple locations can be prioritized on the basis of luminance transients that might serve as a grouping feature. This finding challenges the modal view that transient-based prioritization is limited to four objects (Burkell \& Pylyshyn, 1997; Yantis \& Jones, 1991). In addition, the present results suggest that prioritization by luminance transients might play an important role in the preview benefit (Donk \& Theeuwes, 2001; Peterson, Belopolsky, \& Kramer, 2003). Further research is needed to find out whether such prioritization is produced by bottom-up attentional capture or by temporal grouping.

\section{REFERENCES}

Burkell, J. A., \& Pylyshyn, Z. W. (1997). Searching through subsets: A test of the visual indexing hypothesis. Spatial Vision, 11, 225-258.

Donk, M., \& Theeuwes, J. (2001). Visual marking beside the mark: Prioritizing selection by abrupt onsets. Perception \& Psychophysics, 63, 891-900.

Donk, M., \& Theeuwes, J. (2003). Prioritizing selection of new elements: Bottom-up versus top-down control. Perception \& Psychophysics, 65, 1231-1242.
Egeth, H. E., Virzi, R. A., \& Garbart, H. (1984). Searching for conjunctively defined targets. Journal of Experimental Psychology: Human Perception \& Performance, 10, 32-39.

JiAng, Y., ChUn, M. M., \& MARKs, L. E. (2002). Visual marking: Selective attention to asynchronous temporal groups. Journal of Experimental Psychology: Human Perception \& Performance, 28, 717-730.

Kaptein, N. A., Theeuwes, J., \& van der Heijden, A. H. C. (1995). Search for a conjunctively defined target can be selectively limited to a color-defined subset of elements. Journal of Experimental Psychology: Human Perception \& Performance, 21, 1053-1069.

OLIVERS, C. N. L., \& HuMPhreYs, G. W. (2002). When visual marking meets the attentional blink: More evidence for top-down, limitedcapacity inhibition. Journal of Experimental Psychology: Human Perception \& Performance, 28, 22-42.

Peterson, M. S., Belopolsky, A. V., \& Kramer, A. F. (2003). Contingent visual marking by transients. Perception \& Psychophysics, 65, 695-710.

Pollmann, S., Weidner, R., Humphreys, G. W., Olivers, C. N. L., Müller, K., Lohmann, G., Wiggins, C. J., \& Watson, D. G. (2003). Separating distractor rejection and target detection in posterior parietal cortex: An event-related fMRI study of visual marking. NeuroImage, 18, 310-323.

Theeuwes, J., Kramer, A. F., \& Atchley, P. (1998).Visual marking of old objects. Psychonomic Bulletin \& Review, 5, 130-134.

Treisman, A. M., \& Gelade, G. (1980). A feature-integration theory of attention. Cognitive Psychology, 12, 97-136.

Watson, D. G., \& Humphreys, G. W. (1997). Visual marking: Prioritizing selection for new objects by top-down attentional inhibition of old objects. Psychological Review, 104, 90-122.

Watson, D. G., \& Humphreys, G. W. (2000). Visual marking: Evidence for inhibition using a probe-dot detection paradigm. Perception \& Psychophysics, 62, 471-481.

WATSON, D. G., \& HuMPHREYS, G. W. (2002). Visual marking and visual change. Journal of Experimental Psychology: Human Perception \& Performance, 28, 379-395.

YANTIS, S., \& JoNES, E. (1991). Mechanisms of attentional selection: Temporally modulated priority tags. Perception \& Psychophysics, $\mathbf{5 0}_{2}$ 166-178.

(Manuscript received June 26, 2003; revision accepted for publication March 17, 2004.) 